\title{
An Analysis on the Application of Micro Lecture of Grammar Teaching in TCFL and the Thinking on Its Development
}

\author{
Wang Ruifeng \\ Beijing Language and Culture University \\ Beijing, China
}

\begin{abstract}
The paper analyzes the existing micro lecture of Chinese grammar teaching as a foreign language and its problems from the teaching content, teaching means, basic stricture and supporting resources and puts forward four suggestions on the future development of the micro lecture of Chinese grammar teaching.
\end{abstract}

Keywords-Micro Lecture; Chinese Grammar; TCFL

\section{INTRODUCTION}

Since the American scholar David Penrose proposed the concept of micro lecture in 2008, the academic circles have devoted a lot of enthusiasm and energy to the research and practice of it. However, the opinions vary on the understanding of different aspects of micro lecture, and no unanimous conclusion can be drawn on the length of micro lecture, the form of its teaching video, learning context, learning terminal and course system. ${ }^{1-7]}$ We agree with Hu Tiesheng's definition on micro lecture: micro lecture is a new type of contextualized online video course which supports various ways of learning with the micro teaching video as the main carrier. It is designed and developed for the knowledge points or teaching links of a certain discipline. With micro video as the core, it includes micro teaching plan, micro exercise, micro courseware, micro reflection, micro feedback, micro comment and other supporting and extensible resources, so as to form a semistructured, web-oriented, open and contextualized resource dynamic creation and interactive teaching application environment.

In recent years, all kinds of educational units, professions and areas in China have been actively constructing and applying micro lecture. The research on the micro lecture of teaching of Chinese as a foreign language (TCFL) is still little in the circle of Chinese international education. As of July 2016, there have been only three studies on it: Macro Thinking of the Application of Micro Lecture in Chinese Teaching ${ }^{[8]}$, Design of the Micro Lecture of Chinese Character Teaching ${ }^{[9]}$, Design of the Micro Lecture of Culture Teaching ${ }^{[10]}$. In general, only a few studies have been conducted on the micro lecture of TCFL, and most of them are the macro thinking on the theoretical stage of design thinking without implementation and reflection.

With the existing online micro lectures of TCFL as the

Supported by Science Foundation of Education reform project of BLCU(AZ201503) research objects, the paper focuses on analyzing and studying the current status and existing problems of the micro lectures of Chinese teaching, especially Chinese grammar teaching, and then discussing the development trend of the micro lecture of Chinese grammar teaching on this basis, in order to provide ideas and support for the construction of the micro lecture of Chinese grammar teaching in the future.

\section{ANALYSIS OF THE MiCRo LECTURE OF GRAMMAR TEACHING IN TCFL}

We have searched the main micro lecture and Massive Open Online Course (MOOC) platforms at home and abroad with Chinese, grammar and TCFL as the keywords. As of January 2017, a total of three micro lectures of TCFL have been searched: Chinese Grammar Class(CGC), Extensive Course in Chinese Grammar(ECCG) and Micro Lectures of Basic Chinese Grammar(BCG). It can be seen from the results that the micro lecture of grammar teaching in TCFL is still in the trial and budding stage. Compared with the thousands of TCFL units in China, only three micro lectures are really too few. However, it must be admitted that there may be some micro lectures which are not on the searched platforms or which have been in production but have not yet been uploaded.

The existing micro lectures of grammar teaching in TCFL all take the basic Chinese grammar as the teaching content. Each micro lecture teaches a grammar point. From the nature and quantity of the grammar point, those three micro lectures are slightly different. The micro lecture of ECCG makes micro courses for several basic grammar points in the primary Chinese respectively. At present, there are 5 micro courses on line. Like ECCG, BCG also teaches the basic grammar points of the primary Chinese, but it involves more grammar points. At present, there are 21 grammar points on line. Different from two micro lectures above, the grammar points in CGC all have complicated meanings and usage. Each grammar point has a series of micro courses containing 3 to 6 lectures. From the grammar points taught in the micro lectures above, those three micro lectures have their own characteristics. BCG covers a large quantity of grammar points, so it is more complete, but its internal systematisms is poor. Its 21 grammar points are neither the most basic or important ones nor interacted between each other. The ECCG also has the same problem, and it covers too few grammar points. CGC focuses on the complicated grammar points, in which several micro courses of each 
grammar point systematically teaches its definitions and usage, reflecting strong systematisms and relevance. In addition to grammar points, some micro lectures also integrate the teaching of culture points. The micro lecture of ECCG and BCG just focus on the grammar points, but those of Chinese Grammar Class also blend the culture points into the teaching of grammar points purposefully. The webpage of the platform gives picture, Chinese and English explanations for those culture points.

From the teachers' teaching means in the micro lectures, the micro lectures of existing Chinese grammar teaching mainly combine scene video, animation and picture. The micro courses in some micro lectures adopt the unified means. For example, BCG all combines with animation in teaching. Some micro courses adopt various means.

From the basic structure of each micro course in the micro lecture, some micro courses have the same structure. For example, each micro course in BCG follows the following structure: first of all, the teacher introduces and explains the grammar points based on the animation videos (3 to 4 animation videos); secondly, the teacher summarizes the basic structure and characteristic of the grammar point based on the examples; finally, some practices will be introduced under the guidance of the animation videos. The second batch of on-line micro courses also adds the scene video part to present the real life scene of the grammar point. However, the other two micro courses have diversified structures. The basic structure of some micro courses in ECCG is: the teacher teaches and practices the grammar points based on the scene videos first and then summarize the basic structures and characteristics; in some micro courses, the teachers first introduce the grammar points based on the scene videos, then teach the grammar points based on the animation videos, and finally summarize the basic structures and characteristics. In some micro courses of CGC, the teachers first teach the grammar points based on the sentences in the scene videos, and then summarize the basic structures and examples; in some micro courses, the teachers first teach the grammar points based on the animation, then summarize the basic structures and characteristics, and finally give some practices under the guidance of the animation.

From the supporting resources, in addition to the teaching videos, the micro lectures above also have some supporting resource on the webpage of the platform. The web resources of those three micro lectures are mainly in the following forms: "structure and examples", test yourself, and culture point interpretation.

\section{EXISTING Problems IN THE MiCRO LeCTURES OF GRAMMAR TEACHING IN TCFL}

Some positive and meaningful explorations and experiences have been accumulated in the construction of the micro lectures of Chinese grammar points, and the direction and way of micro lecture construction are gradually clear, which lays a solid foundation for the micro lecture construction of Chinese grammar in the future.

However, the micro lecture is a new thing. The micro lecture construction is still not mature in the developed countries, and it is just in infancy in China. For international education of Chinese, most of the teachers have the Chinese language and literature and linguistics background. They mainly focus on the language ontology and the second language acquisition with neither attention to the fields of education and information science and nor understanding of the new changes and reforms in those two fields. Because of these, the micro lecture construction of Chinese grammar points has the following prominent problems at present:

(1) The whole micro lectures lack the overall and systematic top design and the coordination among team members. The micro courses are scattered, random and not systematic. It is mainly manifested in the following two aspects: first of all, the selection of grammar points is casual. The grammar points in three micro lectures of grammar mentioned above are selected from the primary Chinese grammar, but the selection principle seems to be disorganized and random, neither in accordance with the difficulty nor on the basis of the importance and commonness of the grammar points. Secondly, the micro courses in the same micro lecture are disorganized in structure and content. As already analyzed, the structures and contents in Extensive Course of Chinese Grammar and CGC are not unified. Each micro course shows prominent natures, leading to the poor integrity. For the unity or diversity of the structure and content of the micro courses in the same micro lecture, different understandings can be obtained from different perspectives. "Unity" makes the whole micro lecture system show strong integrity; from another point of view, however, it seems to be single without changes. "Diversity" makes the micro courses rich and changeable, but the whole micro lecture system seems to lack the integrity. It is believed that a perfect micro lecture system should show diversity and richness internally and present integrity overall. However, it is important to note that the internal diversity and richness should not be caused by randomness or lack of coordination, but be intentionally planed after fully studying what kind of grammar point suits for what kind of form and structure.

(2) The micro lectures do not give the learning path instructions. Imagine that a learner opens the web page of a micro lecture and sees so many micro courses of grammar points on it. He must be at a loss as to learn which one first. For this problem, the micro lecture designers do not specify it clearly on the platform. Since the Chinese grammar points are interconnected with each other with different levels of difficulty, the learning order is very important for learners. Without such an instruction, they will feel confused and discouraged. Then, the learning effect will be poor.

(3) The micro lectures lack enough supporting resources. Micro video is the main carrier and form of manifestation of the micro lecture, but it is not all of micro lecture. In addition to the teaching video, the micro lecture needs also sufficient supporting resources on the platform to play a bigger role. From the supporting resources of the micro lecture platform of TCFL, there are only teaching courseware, structure and examples, test yourself, situational dialogues, culture point interpretation, etc. And, most of the micro lectures have only one or two support resources on the platform.

(4) The micro lectures lack interaction. The main objective of language teaching is to develop the language communication 
skill. It needs to give the learners a large number of opportunities to make verbal communication and interaction practices. However, the micro lecture platforms fail to provide such verbal communication and interaction opportunities. The learners can generally watch the micro lecture video on the platform, view the contents on the web page, do the exercises and then receive the feedback given by the system. They cannot communicate with the teachers and get timely help if they have a question. Moreover, the system just gives the feedback of the choice question, true or false item and other objective questions without the practice and examination of subjective questions. The micro course "subject-predicate predicate sentence" in Extensive Course of Chinese Grammar designs the subjective question, such as "describing your family members by the subject-predicate predicate sentence. It is very suitable for practicing and examining the grammar point, but the teacher cannot monitor and evaluate the learner's output sentence.

\section{THINKING ON THE DEVELOPMENT OF THE MiCRO LECTURE OF GRAMMAR TEACHING IN TCFL}

To solve the existing problems in the micro lectures of grammar teaching in TCFL mentioned above, it needs to do the following aspects of work:

(1) Making the overall and systematic top design on the series of micro lectures of Chinese grammar, and designing, developing and applying the micro lectures from the perspective and altitude of curriculum. As $\mathrm{Hu}$ Tiesheng ${ }^{3]}$ points out, "the curriculum attribute should be the root attribute of the micro lecture. Otherwise, the micro lecture can only act as a role of resource bundle. The development of educational informatization in China for many years just proves that the pure resources cannot bring the revolutionary effect on education, no matter how exquisite they are. Therefore, only by designing, developing and applying the micro lectures from the perspective and altitude of curriculum can the micro lecture develop further and better and really change our existing education teaching methods and improve the education teaching quality."

To do this job well, the first thing is to select and determine the grammar points on the basis of ontology research and teaching research. It is particularly important to think about the difficulty and complexity of the grammar points and determine how many micro courses are made for each grammar point. For example, some grammar points are very easy and simple, such as expression of time, so one micro lecture can be made; some are very difficult or complicated, so it needs to determine how many micro courses to produce.

The second is to determine the most appropriate way of introducing, explaining and practicing the micro courses of each grammar point. It needs to discuss with the experts of grammar research and teaching based on the in-depth study of the ways of micro lecture production at home and abroad to determine the way of micro lecture production of each grammar point.

The last is to sort these grammar points scientifically and determine the sequence and hierarchy. This kind of series of micro lectures can provide the learners with a knowledge map, which means making the scattered knowledge pints associated with each other to form a web map, so as to ensure the association and production. The knowledge map can show the learning path for the learners and help them make the study schedule according to their own demands, learn the knowledge points from easy to difficult and clearly point out the skills required, so as to make the learners know their learning tasks and avoid the knowledge fragmentation and isolation. The learner's learning path can also be determined on the basis of the learning analysis technique. The micro lecture platform has acquired and stored a large amount of data about the learners' complex learning behaviors during the learning process, including interested resources, learning time, clicking record, etc. Those data can be regarded as the basis for the platform to recommend the learning path, automatically push the learning resources and teachers for the more targeted teaching. The learners can also see their learning process in the navigation after logging on to the platform every time to review what they have learnt. The system will also recommend the next suitable micro lecture in accordance to the principle of learning from easy to difficulty, so as to make the learners improve step by step.

(2) Strengthening the overall arrangement of the micro lecture production team and the coordination among the members. According to the previous analysis, the selection of grammar points in the micro lectures of Chinese grammar teaching is random, and the structures and contents of micro courses in the same micro lecture are disorganized and inconsistent. In addition, the forms of subtitles of some micro courses in the same micro lecture are inconsistent. Some micro courses have the Chinese, English and pinyin subtitles for the teachers' words and the dialogues in the scene videos and animation videos. Some just have the Chinese and pinyin subtitles or have no subtitle. This is because the existing micro lectures of Chinese grammar mostly adopt the individual contract system. After obtaining the program, the team members select the grammar points and produce the corresponding micro courses independently. The teachers design the micro courses according to their own understandings and habits, so there is neither overall arrangement nor discussion and coordination among the members. This kind of "individual combat" causes the disorganization in the teaching means, structures, contents and subtitles of the micro courses in one micro lecture. In addition, the "individual combat" cannot guarantee the production quality of each micro course. In other words, the teaching design idea of a teacher is not likely to be the most appropriate and scientific. To solve these problems, it needs to change the "individual contract system" into the "the collective cooperation system”. It means all team members discuss with relevant specialists to determine the overall design and details of the micro lecture and give full play to the join forces to make the production of each micro course based on the collective wisdom. It can well ensure the systematisms and quality of the micro lecture. In comparison, among three micro lectures of Chinese grammar, the micro courses in BCG are unified in both the teaching means and structure. This is because it does well in overall organization and coordination. The whole team has held several meetings to discuss selection of grammar points, teaching means and structure of micro courses, basic format of the script and even subtitle format of 
the micro course video, the teacher' s opening and concluding remarks, so as to ensure the systematisms and integrity of the whole micro lecture.

(3) Improving the supporting resources of the existing micro lectures of Chinese grammar and making each micro lecture become a complete resource system. The content development of the micro lecture is a complicated system project. As mentioned above, it is not just a teaching video clip, but also a complete contextualized and interactive resource system including the teaching design, materials, courseware, practice, test, student' $\mathrm{s}$ feedback, teaching reflection, teacher' s comment and interaction between teachers and students related to the grammar point. According to the previous analysis, the supporting resources of the existing micro lectures of Chinese grammar teaching contain only the teaching materials, courseware (teaching courseware, structure and examples, situational dialogue and culture point explanation) and practice and test (test yourself).

(4) Strengthening the interactivity of the micro lectures. According to the previous analysis, the existing micro lectures of Chinese grammar lack the interaction, which is a great defect for language learning specially needing interactive communication. Some good methods of foreign micro lectures and MOOC in promoting interaction are worth learning. To solve the assessment on the subjective questions, Coursera platform adopts the way of mutual-assessment by the learners. The learners can evaluate and give feedback on others' assignments. Research has found that this way of peer assessment can promote the communication between learners and improve their learning motivation and engagement.

Some courses also set the bulletin board system (BBS). The learners can ask questions to the teachers or other learners. However, BBS has two prominent problems: first of all, it is difficult for the teacher to answer all problems of thousands of course learners. To solve this problem, Coursera platform provides the learners with BBS assistant to communicate with them and answer their academic questions and deal with their technical problems. Edx platform provides every learner with a teaching guidance counselor to make the real-time communication through Skype and other social software, so as to grasp their learning conditions and answer the questions. Secondly, the degree of participation in the interaction of language course BBS is not high. The using situation of the existing Chinese MOOC BBS obviously reflects this problem. For example, in the BBS of the Chinese MOOC Chinese for Beginners, most students just say "Hi/Hello, I'm XX". The learners of Intermediate Chinese Grammar have greater Chinese language ability, but in its BBS, the number of questions is small, and the interaction is poor, let alone peer interaction. Most of the members in the BBS rarely post or reply. It may be caused by the one-way input nature of the language course and the learners' limited language proficiency.

Some lectures adopt the offline activities to increase the interactivity. For example, Future learns platform holds small offline meetings regularly to make up for the sense of distance between teachers and learners caused by the network teaching.
Irrational Behavior opened by Duke University records the learners' questions and give feedback in the form of discussion.

The discussion based on interaction above is carried out from the perspective of taking the micro lecture of Chinese grammar as the independent online course and realizing the learners' autonomous learning. If the micro lecture of grammar teaching is defined to be one part of the blended learning which is combined with the classroom teaching and the content for students to preview before class or review after class, the teaching will become a flipped classroom, in which the interaction between teachers and students can be made up.

\section{DISCUSSION}

In the era of rapid development of new information technology, we must "pay attention to the current status and trend of the educational technology in the world and carry out the Chinese teaching-oriented educational technology theory and practice research, which will promote the Chinese language teaching to move forward in the information age. It is also the need of the international education in Chinese for vigorous development.",[13]

The systematic design and construction of the micro lecture system of Chinese grammar teaching is the necessary requirement and development state for the micro lecture of Chinese grammar teaching from the initial randomness to the systematic and scientific natures. The research will also promote the micro lectures of Chinese grammar teaching to become mature and provide the beneficial reference and strong support for the development of the micro lectures of the second language grammar teaching.

\section{REFERENCES}

[1] Tian Qiuhua. "Micro Lecture and Its Development Strategy," Curriculum, Teaching Material and Method, vol.5, 2009. (In Chinese).

[2] Hu Tiesheng, Huang Mingyan, Li Min. "Three Stages of Micro Lecture Development in China and Its Enlightenment,” Journal of Distance Education, vol.4,2013. (In Chinese).

[3] Hu Tiesheng. "Recognition of the Attributes of Micro-lecture and Suggestions on Its Development,” Information technology Education in Primary and Middle Schools, vol.10, 2014. (In Chinese).

[4] Li Yuping. "Micro Lecture---Walk towards Simple Learning," China Information Technology Education, vol.11, 2012. (In Chinese).

[5] Li Jiahou. "The Meaning and Development of Micro Course," Information technology Education in Primary and Middle Schools, vol.3, 2013. (In Chinese).

[6] Jiao Jianli. "Micro Course and Its Application and Influence," Information technology Education in Primary and Middle Schools, vol.4, 2013. (In Chinese).

[7] Liang Leming, Liang Jinming. "From Resource Construction to Application: the Current Status and Application of Micro-lecture," China Educational Technology, vol.8, 2013. (In Chinese).

[8] Xu Yan. "Research on the Application of Micro-lecture in TCFL," New Campus (Print of the First Ten Days of A Month), vol.3, 2016. (In Chinese).

[9] Yu Jia. Design and Development of the Micro Lectures of Character Evolution in TCFL. Kunming, Yunnan. Yunnan Normal University, 2015. (In Chinese). 
[10] Xiong Sang. Research on the Design of the Micro Lectures of Culture in TCFL ---Take Chinese Knot as An Example. Jinan University, 2015. (In Chinese).

[11] Wang Tianmiao, Pei Bojie. “Case Study of Chinese MOOC,” Journal of Research on Education for Ethnic Minorities, vol.2, 2016. (In Chinese).
[12] Xu Jingning. "Thinking based on the MOOC of Intermediate Chinese Grammar," China University Teaching, vol.4, 2016. (In Chinese).

[13] Zheng Yanqun. "Enlightenment of the Trend of the World's Educational Technology on Chinese Teaching," Chinese Teaching in the World, vol.2, 2013. (In Chinese). 